\title{
Lexically independent priming in online sentence comprehension
}

\author{
MatThew J. Traxler \\ University of California, Davis, California
}

\begin{abstract}
Two eye-tracking experiments investigated what happens when people read pairs of sentences that have the same syntactic structure. Previous experiments have shown priming in online sentence processing only when critical lexical material overlaps between the prime and the target sentence. In the current study, participants were asked to read sentences containing modifier-goal ambiguities. Half of the target sentences were preceded by sentences with the same structure, and half were preceded by sentences with a different structure. In Experiment 1, the prime-target pairs had the same main verb. In Experiment 2, the prime-target pairs had different main verbs. Facilitated target sentence processing was observed in both Experiments 1 and 2 when the target sentences were preceded by a prime sentence with the same syntactic structure. These results provide the first evidence of lexically independent, between-sentence structural priming in online sentence comprehension.
\end{abstract}

When comprehenders parse sentences, they determine how words relate to one another. How do comprehenders determine these relationships? One challenge that comprehenders must overcome to parse sentences is determining whether words are related as arguments or as adjuncts. Arguments are obligatory semantic elements of an expression that are usually overtly realized in the syntax. Adjuncts are optional elements at both levels. As Jackendoff (2002, pp. 132-134) explains, the verb devoured must have two arguments: a subject and an object. The sentence "John devoured," for example, is ungrammatical. By contrast, a semantically similar verb, ate, requires only one syntactically realized argument-a subject, as in "John ate"-although the object of ate may be semantically required if, for instance, the sentence were interpreted as saying "John ate something." All verbs allow, but do not require, various types of semantic modifiers, such as locatives, temporals, indications of manner, and so on. These optional semantic elements are often realized as syntactic adjuncts. So, for example, "John ate a sandwich at the cafe at three o'clock with gusto before the meeting" contains four adjuncts of ate: "at the cafe," "at three o'clock," "with gusto," and "before the meeting."

This article tries to tease apart different theories that explain how comprehenders parse sentences. Autonomous syntax approaches (e.g., Frazier, 1979) appeal to general processing strategies, or heuristics, to explain how comprehenders establish connections among words. Lexicalist approaches (e.g., Jackendoff, 2002; MacDonald, Pearlmutter, \& Seidenberg, 1994) propose that structural information is tied to specific words. Still other approaches suggest that only some of the structural information necessary to establish dependencies between words is lexically stored. For example, some accounts propose that structural information about arguments is lexically stored, but other kinds of structural relations are derived by rule. One such account is the argument structure hypothesis (Boland \& Blodgett, 2006), which proposes that information about syntactic structures is lexically stored and retrieved for arguments, but that adjunct relationships are computed de novo whenever they appear in the input. Because information relating to arguments is stored and used differently than information relating to adjuncts, the argument structure hypothesis predicts that readers will respond to arguments and adjuncts in different ways.

This prediction is supported by a number of online parsing studies. Comprehenders include semantically required but syntactically optional arguments in their representations of sentences (Koenig, Mauner, \& Bienvenue, 2003; Mauner, Tanenhaus, \& Carlson, 1995). Further, if a phrase is temporarily ambiguous between an argument and an adjunct, it takes longer to process when the correct interpretation requires it to be an adjunct (Clifton, Speer, \& Abney, 1991; Foraker \& McElree, 2001; Liversedge, Pickering, Branigan, \& van Gompel, 1998). Boland and Blodgett (2006) extended this principle to arguments of nouns. They showed that arguments of both verbs and nouns were processed more easily than were adjuncts. They concluded that structural information about arguments is stored in argument structure representations and is activated during lexical access. Adjunct processing, by contrast, is governed by "non-lexicalized syntactic rules" (p. 388).

Further evidence that argument structure information affects online sentence comprehension has been obtained using the syntactic priming paradigm. In previous comprehension priming studies involving argument relations,

M. J. Traxler, mjtraxler@ucdavis.edu 
priming effects were much larger when the same verb appeared in both the prime and target sentence, and priming may not have occurred otherwise (Arai, van Gompel, \& Scheepers, 2007; Pickering \& Traxler, 2004; see also Ledoux, Traxler, \& Swaab, 2007). In some of these experiments, a target sentence like (1) was preceded by a prime sentence like (2) or (3):

(1) The defendant examined by the lawyer was guilty.

(2) The engineer examined by the board passed with flying colors.

(3) The engineer tested by the board passed with flying colors.

Prime sentences like (2) led to speeded processing of the syntactically disambiguating "by" phrase. Prime sentences like (3) did not noticeably affect processing of the disambiguating "by" phrase. Because priming in comprehension is larger with verb overlap and potentially absent without it, it may reflect lexical projection of the syntactic structure used to determine how defendant and lawyer relate to examined in sentences like (1).

The current study was designed to test whether similar priming effects can be observed without lexical overlap for nonargument relations. Although lexically independent effects have been obtained with other sentence types in production (e.g., Bock, 1986), in offline interpretation-choice tasks in comprehension-tocomprehension priming (Branigan, Pickering, \& McLean, 2005), within sentences for coordinate structures (Frazier, Munn, \& Clifton, 2000), and for subcategorization frames in a fast-priming paradigm (Trueswell \& Kim, 1998), such effects have not been observed between sentences in an online comprehension task. In these experiments, the relevant structural decisions involve adjunct relations; lexically independent priming effects would be consistent with Boland and Blodgett's argument structure hypothesis as well as with constraint-based accounts that propose a graded, rather than categorical, distinction between arguments and adjuncts (MacDonald et al., 1994).

\section{EXPERIMENT 1}

Test sentences resembled target Sentence 1c below (see Table 1): 1a. The girl tossed the blanket on the bed into the laundry this morning. (same-structure prime)

1b. The girl tossed the blanket into the laundry this morning. (different-structure prime)

1c. The vendor tossed the peanuts/ in the box/ into the crowd/ during the/ game. (target)

Slashes indicate where the sentences were segmented for analysis.

The test sentences are temporarily ambiguous with respect to which preceding element they modify. The first can be interpreted as an argument, as in, "The vendor tossed the peanuts in the box just now," but it turns out to be an adjunct of the preceding noun peanuts, as in, "Which peanuts?" "The peanuts in the box." Usually, comprehenders experience difficulty processing the second prepositional phrase (PP) into the crowd, because the preceding PP has been interpreted as an argument and must be displaced by the second PP. If the adjunct interpretation of the first PP can be primed, then reading two modifier-goal sentences (such as 1a and 1c) in a row should lead to facilitated processing of the second PP in the target sentence. If both adjunct and argument relations are lexically governed (as in MacDonald et al., 1994), then more priming should occur when the prime and target sentences have the same verb. In Experiment 1, the same verb always appeared in both the prime and the target sentence. In Experiment 2, the prime and target sentences had different verbs.

\section{Method}

Participants. Forty-eight native English speakers with normal vision and hearing participated for course credit.

Items. The items were 40 prime-target pairs, similar to Sentences $1 \mathrm{a}-\mathrm{c}$ above. The stimuli are available from the author. The target sentences were always ambiguous modifier-goal sentences like Sentence 1c. Half of the primes had the same structure as the target (as in 1a). The other half of the primes had only an argument PP (as in 1b).

The items were randomly assigned to four lists so that (1) equal numbers of each kind of prime-target pair appeared on each list, (2) participants were exposed to only one version of each sentence, and (3) presentation order of different prime-target combinations was counterbalanced. Each target from one list served as a prime on another list.

Participants also read 64 filler items of various types. At least one filler item intervened between each prime-target pair. Less

Table 1

Sample Stimuli for Experiments 1 and 2

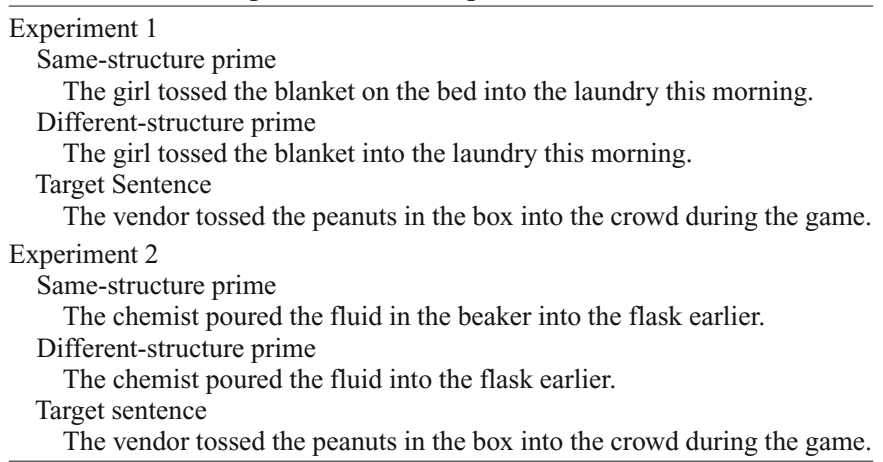


Table 2

Mean Scores (in Milliseconds) on the Dependent Measures by Region and Condition for Experiment 1

\begin{tabular}{llcccc}
\hline & & \multicolumn{3}{c}{ Dependent Measure } \\
\cline { 3 - 6 } \multicolumn{1}{c}{ Region } & \multicolumn{1}{c}{ Condition } & $\begin{array}{c}\text { First Pass } \\
\text { Time }\end{array}$ & $\begin{array}{c}\text { \% First Pass } \\
\text { Regressions }\end{array}$ & $\begin{array}{c}\text { Regression } \\
\text { Path Time }\end{array}$ & $\begin{array}{c}\text { Total } \\
\text { Time }\end{array}$ \\
\hline \multirow{2}{*}{ First PP } & Baseline & 446 & 8.1 & 482 & 600 \\
& Same structure & 444 & 12.7 & 500 & 589 \\
\multirow{2}{*}{ Second PP } & Different structure & 425 & 7.9 & 460 & 592 \\
& Baseline & 502 & 18.4 & 568 & 689 \\
\multirow{3}{*}{ Postdisambiguation } & Same structure & 489 & 14.7 & 584 & 666 \\
& Different structure & 503 & 15.2 & 601 & 733 \\
& Baseline & 360 & 36.3 & 586 & 469 \\
& Same structure & 375 & 27.4 & 531 & 448 \\
& Different structure & 371 & 37.5 & 598 & 470 \\
\hline
\end{tabular}

than one third of the total number of sentences were ambiguous modifier-goal sentences.

The baseline condition was based on the eye-movement record as participants read the modifier-goal primes. The baseline condition indexes the effects that the critical sentence has when it is preceded by unrelated material. The same-structure condition was based on eye movements as participants read modifier-goal targets following modifier-goal primes. This condition assesses the benefit of having a target sentence with the same main verb and syntactic structure as the prime. The different-structure condition was based on eye movements as participants read modifier-goal targets following goal primes. Comparing processing in the samestructure and different-structure conditions provided another estimate of priming.

Procedure. A Fourward Technologies Dual Purkinje Image Eye Tracker monitored participants' eye movements. The tracker has angular resolution of 10' of arc. It monitored only the right eye. A PC displayed materials on a monitor $70 \mathrm{~cm}$ from the participants' eyes. The display consisted of Borland $\mathrm{C}$ default font with four characters per degree of visual angle. The location of participants' gaze was sampled every millisecond, and the software recorded the tracker's output to establish the sequence of eye fixations and their start and finish times. The experimenter used a bite plate and head rests to minimize participants' movements. After the tracker was aligned and calibrated, the experiment began. After reading each sentence, participants pressed a key. Participants responded to a comprehension question after 12 of the sentences, but never after a prime. Participants did not receive feedback. Calibration checks occurred between trials, with recalibrations made as necessary.

\section{Results}

Table 2 presents means for four standard dependent measures by scoring region and condition. The adjunct $P P$ region consisted of the first prepositional phrase. The disambiguating $P P$ region consisted of the second postverbal PP. The postdisambiguation region consisted of two words immediately following the disambiguating PP.

First-pass time is the sum of all fixations, beginning with the first fixation in a region and ending when the reader's gaze leaves the region. First-pass regressions is the number of eye movements that crossed a region's left-hand boundary immediately following a first-pass fixation. Regressionpath time includes all fixations, beginning with the first fixation in a region and ending when the reader's gaze crosses the right-hand boundary. Total time is the sum of all fixations in a region. All fixation times less than $120 \mathrm{msec}$ or greater than 3,000 msec were excluded, eliminating 7.8\% of the data. Any fixation times greater than 2.5 standard deviations from the by-subjects condition mean were also deleted, eliminating $8.1 \%$ more of the data.

Data from each region were separately submitted to $1 \times 3$ (condition: baseline vs. same-structure vs. different-structure) repeated measures ANOVAs with participants and items treated as random factors. Statistics computed on the first-pass regressions data are based on arcsine transformed proportions. Table 3 lists statistically significant outcomes. The abbreviation prep represents the probability-of-replication statistic (Killeen, 2005), and $d$ is an effect size estimate for the means comparisons (Glass \& Hopkins, 1984, p. 373).

First-pass regressions and regression-path time differed across conditions in the adjunct region. The same-structure condition had more first-pass regressions than either the baseline condition $\left[12.7 \%\right.$ vs. $8.1 \% ; F_{1}(1,47)=4.68$, prep $=.917, d=.44 ; F_{2}(1,39)=6.03$, prep $=.95, d=$ $.55]$ or the different-structure condition $[12.7 \%$ vs. $7.9 \%$; $F_{1}(1,47)=7.20$, prep $=.966, d=.54 ; F_{2}(1,39)=6.26$, prep $=.966, d=.56]$. Regression-path results also indicated greater processing time in the same-structure condition than in the different-structure condition $\left[F_{1}(1,47)=\right.$ 13.4 , prep $=.99, d=.76 ; F_{2}(1,39)=7.50$, prep $=.966$, $d=.62]$. More regressions and higher regression-path time in the repeated structure condition may indicate that readers initiated syntactic reanalysis earlier when structure was repeated across the prime and target sentences than when structure was not repeated.

Table 3

Results for the $1 \times 3$ (Baseline vs. Same-Structure vs. Different-Structure Condition) Omnibus ANOVAs by Region for Experiment 1

\begin{tabular}{lcccccc}
\hline & $F_{1}$ & prep & $M S_{\mathrm{e}}$ & $F_{2}$ & prep & $M S_{\mathrm{e}}$ \\
\hline $\begin{array}{l}\text { First PP } \\
\quad \text { First pass regressions }\end{array}$ & 4.58 & .966 & 0.008 & 4.76 & .966 & 0.006 \\
$\quad \begin{array}{l}\text { Regression-path time } \\
\quad\end{array}$ & 6.73 & .986 & 2,739 & 3.84 & .917 & 3,406 \\
$\begin{array}{l}\text { Second PP } \\
\quad \text { Total time }\end{array}$ & 5.99 & .966 & 9,338 & 4.56 & .966 & 10,713 \\
$\quad \begin{array}{l}\text { Postdisambiguation } \\
\quad \text { First pass regressions }\end{array}$ & 8.55 & .990 & 0.017 & 3.83 & .917 & 0.029 \\
$\quad \begin{array}{l}\text { Regression-path time } \\
\quad \text { Total time }\end{array}$ & 6.64 & .966 & 9,315 & 5.93 & .966 & 12,800 \\
& 1.62 & .818 & 4,720 & 3.13 & .917 & 5,073 \\
\hline
\end{tabular}

Note-Degrees of freedom for condition effect are $(2,94)$ by participants and $(2,78)$ by items. prep, probability of replication. 
Table 4

\begin{tabular}{llcccc}
\multicolumn{5}{c}{$\begin{array}{c}\text { Mean Scores (in Milliseconds) on the Dependent Measures by } \\
\text { Region and Condition for Experiment 2 }\end{array}$} \\
\cline { 3 - 6 } & & \multicolumn{4}{c}{ Dependent Measure } \\
\cline { 3 - 6 } \multicolumn{1}{c}{ Region } & \multicolumn{1}{c}{$\begin{array}{c}\text { Condition } \\
\text { First Pass }\end{array}$} & $\begin{array}{c}\text { \% First Pass } \\
\text { Time }\end{array}$ & $\begin{array}{c}\text { Regression } \\
\text { Regressions }\end{array}$ & $\begin{array}{c}\text { Total } \\
\text { Path Time }\end{array}$ & Time \\
\hline \multirow{5}{*}{ Second PP } & Baseline & 450 & 14.5 & 537 & 681 \\
& Same structure & 476 & 10.5 & 537 & 667 \\
\multirow{5}{*}{ Postdisambiguation } & Different structure & 455 & 12.7 & 517 & 673 \\
& Baseline & 469 & 22.1 & 597 & 710 \\
& Same structure & 456 & 23.3 & 607 & 676 \\
& Different structure & 477 & 28.1 & 634 & 745 \\
& Baseline & 378 & 31.1 & 531 & 467 \\
& Same structure & 399 & 32.4 & 577 & 471 \\
& Different structure & 367 & 34.1 & 601 & 489 \\
\hline
\end{tabular}

Total times in the disambiguating region showed that readers had more difficulty with the different-structure condition than with the others. The different-structure condition had greater total time than either the baseline condition $\left[733\right.$ vs. $689 \mathrm{msec} ; F_{1}(1,47)=5.08$, prep $=$ $.917, d=.46 ; F_{2}(1,39)=3.21$, prep $\left.=.878, d=.41\right]$ or the same-structure condition [733 vs. $666 \mathrm{msec}$; $F_{1}(1,47)=11.6$, prep $=.99, d=.69 ; F_{2}(1,39)=9.01$, prep $=.967, d=.66]$ did. Thus, the disambiguating region of the target sentences was harder to process when the prime and target sentences had different structures.

Results from the postdisambiguating region also suggest that ambiguity resolution took longer in the differentstructure condition than in the same-structure condition. Main effects of condition occurred in first-pass regressions and regression-path time. The baseline condition had more first-pass regressions than the same-structure condition did $\left[36.3 \%\right.$ vs. $27.4 \% ; F_{1}(1,47)=12.8$, prep $=$ $.99, d=.73 ; F_{2}(1,39)=4.42$, prep $\left.=.92 . d=.47\right]$. The different-structure condition also had more first-pass regressions than the same-structure condition did [ $37.5 \%$ vs. $27.4 \% ; F_{1}(1,47)=14.3$, prep $=.99, d=.77 ; F_{2}(1,39)=$ 6.93 , prep $=.966, d=.59]$.

In Experiment 1, processing difficulty differed across the baseline, repeated, and different-structure conditions. When the prime and target shared the same structure, processing of the ambiguous region in the target was more difficult, but processing at and immediately following the disambiguating region was easier. When the prime and target had different structures, there was no evidence that reanalysis began prior to the postdisambiguating region. This experiment shows that adjunct interpretation of the first PP can be primed.

\section{EXPERIMENT 2}

Experiment 2 tests whether lexical overlap is necessary for priming in sentences like 1a-c. Experiment 2 was identical to Experiment 1, except that the prime and target sentences were rearranged so that they always had different verbs. Previous eye-tracking studies investigating argument processing have shown that eliminating verb overlap reduces or eliminates priming.

\section{Method}

Participants. Fifty-three people from the same pool as Experiment 1 participated.

Stimuli. The prime and target sentences from Experiment 1 were rearranged so that the verb in the prime sentence was never repeated in the target sentence.

Procedure and Analyses. The eye-tracking procedure, scoring regions, and statistical analyses were identical to those in Experiment 1, and $12.9 \%$ of the data were excluded using the same criteria.

\section{Results}

Table 4 presents mean values of the dependent measures. Table 5 presents the results of the omnibus ANOVAs that were significant by participants, items, or both.

First-pass data from the adjunct PP region indicated greater processing time in the same-structure condition than in the baseline [ 475 vs. $450 \mathrm{msec} ; F_{1}(1,52)=6.29$, prep $=.966, d=.49 ; F_{2}(1,39)=5.43$, prep $=.917$, $d=.52]$ and the different-structure [475 vs. $455 \mathrm{msec}$; $F_{1}(1,52)=4.26$, prep $=.917, d=.39 ; F_{2}(1,39)=4.97$, prep $=.917, d=.50]$ conditions. As in Experiment 1, elevated first-pass times in the modifier PP region suggest that readers began reanalyzing the PP earlier when the prime sentence had the same structure as the target.

In the disambiguating PP region, total time produced a significant condition effect, driven by greater total time in the different-structure condition than the same-structure condition [747 vs. $676 \mathrm{msec} ; F_{1}(1,52)=16.3$, prep $=$ $.99, d=78 ; F_{2}(1,39)=8.09$, prep $\left.=.966, d=.63\right]$. The different-structure condition also had greater total time than the baseline condition [significant by participants $F_{1}(1,52)=4.50$, prep $=.917, d=.41$, but not by items

Table 5

Summary of Significant Statistical Results for the $1 \times 3$ (Baseline vs. Same-Structure vs. Different-Structure Condition) Omnibus ANOVAs by Region for Experiment 2

\begin{tabular}{lcccccc}
\hline & $F_{1}$ & prep & $M S_{\mathrm{e}}$ & $F_{2}$ & prep & $M S_{\mathrm{e}}$ \\
\hline First PP & & & & & & \\
$\quad$ First-pass time & 3.58 & .917 & 2,827 & 3.47 & .917 & 2,514 \\
$\begin{array}{l}\text { Second PP } \\
\quad \text { Total time }\end{array}$ & 8.15 & .990 & 8,231 & 4.04 & .950 & 11,470 \\
$\begin{array}{l}\text { Postdisambiguation } \\
\quad \text { Regression-path time }\end{array}$ & 3.25 & .917 & 20,563 & 2.62 & .878 & 10,447 \\
\hline
\end{tabular}

Note-Degrees of freedom for condition effect are $(2,104)$ by participants and $(2,78)$ by items. prep, probability of replication. 
$F_{2}(1,39)=2.07$, prep $\left.=.818, d=.33\right]$. There also was a trend toward shorter total times in the same-structure condition compared to the baseline in the by-participants analysis $\left[F_{1}(1,52)=3.66\right.$, prep $\left.\sim .90, d=.37\right]$, but not in the byitems analysis $\left[F_{1}(1,39)=1.97\right.$, prep $\left.\sim .82, d=.31\right]$.

The postdisambiguation region also produced some evidence of continuing processing difficulty in the differentstructure condition. Regression-path time was longer in the different-structure condition than in the baseline condition $\left[F_{1}(1,52)=6.31\right.$, prep $=.95, d=.49 ; F_{2}(1,39)=$ 5.20 , prep $=.917, d=.51]$.

Experiment 2 produced a pattern that was qualitatively similar to that of Experiment 1. Readers took longer to process the adjunct PP region in the same-structure condition than in the other two conditions. Reading times at and following the syntactically disambiguating PP region indicated greater difficulty in the different-structure condition than in the baseline or same-structure conditions. These results likely reflect continuing difficulty resolving the ambiguity when the prime sentence had only an argument PP. Thus, priming can occur without lexical or semantic overlap. The fact that priming effects occurred without lexical or semantic overlap points toward a syntactic source of facilitated processing.

\section{COMPARING EXPERIMENTS 1 AND 2}

To determine whether readers undertook qualitatively different processing when the verb in the prime sentence was repeated versus when it was not, combined data were subjected to 2 (verb: repeated vs. new) $\times 3$ (condition: baseline vs. same-structure vs. different-structure) repeated measures ANOVAs. Verb was treated as a betweenparticipants and within-items random factor. Condition was treated as within-participants and within-items factors.

Table 6 presents the significant results for these interaction tests. Figure 1 presents total time results for both ex-
Table 6

Significant Results for ANOVAs Comparing Experiments 1 and 2, Where Source $\equiv$ Verb Overlap

(Verb in the Target Sentence Is Repeated vs. New) $\times$ Condition (Baseline vs. Same Structure vs. Different Structure)

\begin{tabular}{lcccccc}
\hline Region and DV & $F_{1}$ & prep & $M S_{\mathrm{e}}$ & $F_{2}$ & prep & $M S_{\mathrm{e}}$ \\
\hline $\begin{array}{l}\text { Adjunct PP, } \\
\quad \text { First-pass regressions }\end{array}$ & 4.32 & .966 & 0.031 & 4.61 & .966 & 0.035 \\
$\begin{array}{l}\text { Postdisambiguation, } \\
\quad \text { First-pass regressions }\end{array}$ & 3.73 & .950 & 0.030 & 2.14 & .870 & 0.035 \\
$\begin{array}{c}\text { Postdisambiguation, } \\
\quad \text { Regression-path time }\end{array}$ & 4.25 & .950 & 15,223 & 7.43 & .990 & 10,425 \\
\hline
\end{tabular}

periments for the disambiguating PP region, which gives a good indication of overall processing load associated with syntactic disambiguation.

First-pass regressions produced interactions of verb and condition in the adjunct PP and postdisambiguating regions. In the adjunct PP and postdisambiguation regions, the condition effect was significant only when the verbs were repeated. The interaction in the regression-path time data from the postdisambiguation region occurred because, when the verbs were repeated, the same-structure condition had shorter regression-path time than the other two conditions did; but when the verbs were not repeated, only the baseline condition had shorter regression-path time than the different-structure condition did, with the same-structure condition having intermediate regressionpath time.

The interaction tests for the total time data from the syntactically disambiguating second $P P$ region did not indicate a difference between the two experiments $\left[F_{1}(2,198)=\right.$ 0.092 , prep $=.5, M S_{\mathrm{e}}=8,757 ; F_{2}(2,78)=0.022$, prep $\left.=.5, M S_{\mathrm{e}}=12,519\right]$. The main effect of condition was highly significant in the combined data $\left[F_{1}(2,198)=13.9\right.$, prep $=.99 ; F_{2}(2,78)=9.82$, prep $\left.=.99\right]$. Tests for simple effects showed differences among all three conditions. The same-structure condition had shorter total time than both

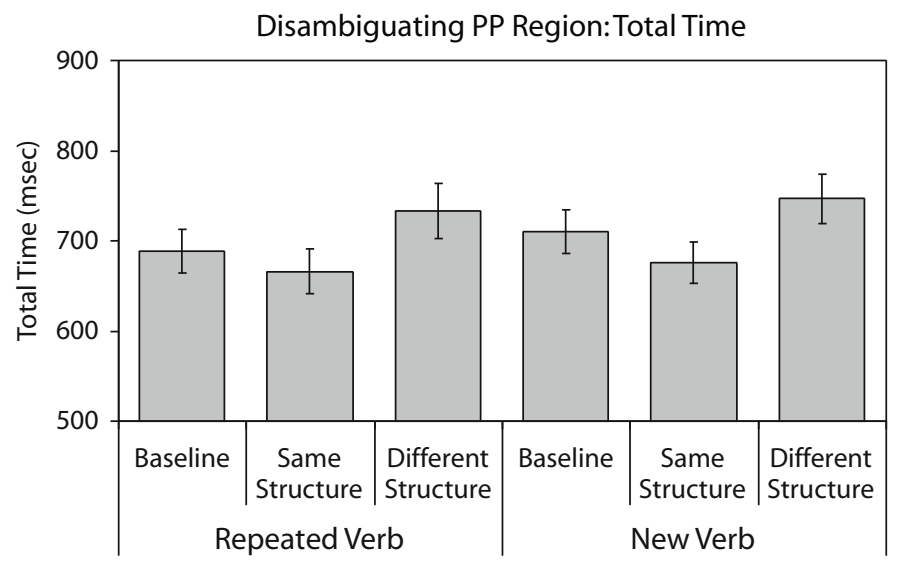

Figure 1. Total time by condition for Experiment 1 (left side) and Experiment 2 (right side) for the syntactically disambiguating region. Overall processing difficulty of the disambiguating region was similar whether the verb was repeated between the prime and target sentence (Experiment 1) or not (Experiment 2). Both experiments show decreased processing difficulty in the same-structure condition as compared with the different-structure condition. Error bars reflect standard errors. 
the baseline condition $\left[671 \mathrm{vs.} 700 \mathrm{msec} ; F_{1}(1,99)=4.66\right.$, prep $=.917, d=31 ; F_{2}(1,39)=3.93$, prep $\left..917, d=.32\right]$ and the different-structure condition $[671 \mathrm{vs} .740 \mathrm{msec}$; $F_{1}(1,99)=27.6$, prep $=.99, d=.74 ; F_{2}(1,39)=19.6$, prep $=.99, d=.70]$. The different-structure condition also had greater total time than the baseline condition did $\left[740\right.$ vs. $700 \mathrm{msec} ; F_{1}(1,99)=9.58$, prep $=.966, d=.43$; $F_{2}(1,39)=5.97$, prep $\left.=.95, d=.39\right]$. As Figure 1 shows, total times for the syntactically disambiguating PP region were remarkably similar whether the verb was repeated or not, suggesting that the overall difference in load when the structure was repeated versus when it was not was about the same whether the verb was repeated or not.

Interactions in first-pass regressions may indicate some subtle differences in early processing when the verb is repeated as opposed to when it is not. In particular, more regressions from the first PP region in the same-structure condition when the verb was repeated might indicate that readers initiated syntactic reanalysis sooner when the verb was repeated. However, there was a significant condition effect in the first-pass fixation data in the same region when the verb was not repeated, with the same overall pattern. Because first-pass time and first-pass regressions are sometimes in a trading relationship (e.g., Rayner, 1998), the first-pass regressions effect in Experiment 1 and the first-pass fixation time effects in Experiment 2 may reflect the same underlying mental process. Specifically, when readers have just read a modifier-goal prime sentence, and they encounter an ambiguous PP in the very next sentence, they initiate reanalysis sooner than they do when a sentence with a different structure precedes the ambiguous target. This conclusion is reinforced by the very similar total time results across the two experiments, especially in the syntactically disambiguating PP region.

\section{GENERAL DISCUSSION}

In Experiment 1, prime and target sentences had the same verb. At the adjunct PP, first-pass regressions and regression-path time were elevated in the same-structure condition, suggesting that reanalysis was initiated during processing of the first prepositional phrase. Total time data from the disambiguating PP and first-pass and regressionpath time data from the postdisambiguating region indicated less processing difficulty in the same-structure condition. In Experiment 2, the sentences were rearranged to eliminate lexical overlap between the prime and target sentences. Early difficulty in the same-structure condition was observed at the adjunct PP, with savings relative to the different-structure condition appearing at the disambiguating PP and in the postdisambiguating region. The pattern of processing difficulty across conditions in the disambiguating region was very similar across the two experiments, as indexed by the total time results. Shorter total times in the same-structure condition than in the different-structure condition (and the baseline in the aggregated data) indicate that the modifier-goal prime sentences were effective and that priming did not depend on verb repetition. The present results do not demonstrate that the pattern of priming was identical across experiments, however, so they do not eliminate the possibility of lexical mediation of priming for the kinds of sentences tested here.

These results comprise the first demonstration of between-sentence priming effects occurring without lexical overlap in online sentence processing. As such, they have a number of theoretical implications. First, it is now fully plausible that previously observed priming effects were not the result of semantic priming or strategic prediction. In the reduced relative and dative-double object alternations, priming in online comprehension has been observed only with lexical overlap. The facilitation could be semantic rather than syntactic. Since priming occurred in Experiment 2, where there was no lexical overlap and the sentence meanings were unrelated, these effects cannot represent a form of semantic priming - at least as semantics is usually defined (see McKoon \& Ratcliff, 2003; Stevenson \& Merlo, 1997, for an alternate perspective). Since no strategic cues other than sentence structure itself were available in the present Experiment 2, priming did not result from readers' predicting the upcoming structure. Hence, the present results likely reflect processes intrinsic to the parser.

The results provide further evidence that different processing principles apply to arguments than to adjuncts. In previous priming studies involving argument relations, syntactic priming has been observed only when verbs were repeated across prime and target sentences. Prior explanations have therefore appealed to lexicalist (e.g., MacDonald, et al., 1994) representation and processing assumptions - namely, that syntactic structures are tied to specific word representations in memory and are activated as words are identified during reading and listening. Previous priming studies might have simply failed to detect lexically independent priming, but it could be that different types of syntactic relations are stored and recovered in different ways. For example, argument relations are considered part of the core meaning of verbs, and verbs differ with respect to the numbers and kinds of arguments they require (Levin, 1993), but adjunct relations are not. Lexically dependent priming for arguments and lexically independent priming of adjuncts could result from differences in the way they are represented and processed. If argument relations are represented in and projected out of verbs' lexical entries, that would explain why argument priming is lexically dependent. If adjunct relations are not lexically represented and are computed based on rules (as Boland \& BoehmJernigan, 1998, and Boland \& Blodgett, 2006, suggested; see also Traxler \& Tooley, 2007), that would account for lexically independent priming for adjunct relations.

How far can these results be generalized? Considerable additional work will be necessary to determine whether lexically independent priming can be observed for other sentence types involving adjuncts. For example, it is not known whether relative-clause attachment decisions can be primed for sentences like "The sister of the mailman who hurt himself/herself was upset." It is also unknown whether lexical repetition will affect the magnitude of priming. For example, would priming effects be larger if the preceding sentence had the word sister modified by a relative clause? This raises a final point about the present 
experiments. The priming effects observed here might have been larger if overlapping nouns rather than overlapping verbs had been used. If the modifier interpretation of the PP were easier to obtain with noun repetition, this might indicate that the necessary structural information is associated with noun representations in the lexicon.

\section{AUTHOR NOTE}

This research was supported by awards from the National Science Foundation (NSF 0446618) and by the National Institutes of Health (R01-HD048914-01A2). The author thanks Kristen M. Tooley for assistance in data collection and three anonymous reviewers for helpful commentary. Correspondence regarding this article should be addressed to M. Traxler, Department of Psychology, University of California, One Shields Avenue, Davis, CA 95616 (e-mail: mjtraxler@ucdavis.edu).

\section{REFERENCES}

Arai, M., van Gompel, R. P. G., \& Scheepers, C. (2007). Priming ditransitive structures in comprehension. Cognitive Psychology, 54, 218-250.

Bock, J. K. (1986). Syntactic persistence in language production. Cognitive Psychology, 18, 355-387.

Boland, J. E., \& Blodgett, A. (2006). Argument status and PP-attachment. Journal of Psycholinguistic Research, 35, 385-403.

Boland, J. E., \& BoeHM-JeRnigan, H. (1998). Lexical constraints and prepositional phrase attachment. Journal of Memory and Language, 39, 684-719.

Branigan, H. P., Pickering, M. J., \& Mclean, J. F. (2005). Priming prepositional phrase attachment during comprehension. Journal of Experimental Psychology: Learning, Memory, \& Cognition, 31, 468-481.

Clifton, C., Jr., Speer, S., \& AbNey, S. P. (1991). Parsing arguments: Phrase structure and argument structure as determinants of initial parsing decisions. Journal of Memory \& Language, 30, 251-271.

Foraker, S., \& McElree, B. (2001, March). The effects of cooccurrence frequencies in the time course of parsing operations. Poster presented at the 14th Annual CUNY Sentence Processing Conference, Philadelphia.

Frazier, L. (1979). On comprehending sentences: Syntactic parsing strategies (Doctoral dissertation, University of Connecticut, 1979). Dissertation Abstracts International, 40, 227A.
Frazier, L., Munn, A., \& Clifton, C., JR. (2000). Processing coordinate structures. Journal of Psycholinguistic Research, 29, 343-370.

Glass, G. V., \& Hopkins, K. D. (1984). Statistical methods in education and psychology. New York: Allyn \& Bacon.

JACKENDOFF, R. (2002). Foundations of language: Brain, meaning, grammar, evolution. Oxford: Oxford University Press.

KilleEn, P. R. (2005). An alternative to null-hypothesis significance tests. Psychological Science, 16, 345-353.

Koenig, J. P., Mauner, G., \& Bienvenue, B. (2003). Arguments for adjuncts. Cognition, 89, 67-103.

LeDoux, K., Traxler, M. J., \& Swaab, T. Y. (2007). Syntactic priming in comprehension: Evidence from event-related potentials. Psychological Science, 18, 135-143.

LEvin, B. (1993). English verb classes and alternations. Chicago: University of Chicago Press.

Liversedge, S. P., Pickering, M. J., Branigan, H. P., \& van GomPEL, R. P. G. (1998). Processing arguments and adjuncts in isolation and context: The case of by-phrase ambiguities in passives. Psychonomic Bulletin \& Review, 24, 461-475.

MacDonald, M. C., Pearlmutter, N. J., \& Seidenberg, M. S. (1994). Lexical nature of syntactic ambiguity resolution. Psychological Review, 101, 676-703.

Mauner, G., Tanenhaus, M. K., \& Carlson, G. N. (1995). Implicit arguments in sentence processing. Journal of Memory \& Language, 34, 357-382.

McKoon, G., \& Ratcliff, R. (2003). Meaning through syntax: Language comprehension and the reduced relative clause construction. Psychological Review, 110, 490-525.

Pickering, M. J., \& Traxler, M. J. (2004, March). Grammatical repetition and garden path effects. Paper presented at the 17th Annual CUNY Sentence Processing Conference, College Park, MD.

RAYNER, K. (1998). Eye movements in reading and information processing: 20 years of research. Psychological Bulletin, 124, 372-422.

Stevenson, S., \& Merlo, P. (1997). Lexical structure and parsing complexity. Language \& Cognitive Processes, 12, 349-399.

TraXler, M. J., \& ToOLey, K. M. (2007). Lexical mediation and context effects in sentence processing. Brain Research, 1146, 59-74.

Trueswell, J. C., \& KIM, A. E. (1998). How to prune a garden path by nipping it in the bud: Fast priming of verb argument structure. Journal of Memory \& Language, 39, 102-123.

(Manuscript received December 9, 2006; revision accepted for publication June 1, 2007.) 